\title{
Temporary Perfusion Branches to Decrease Spinal Cord Ischemia in the Endovascular Treatment of Thoraco-Abdominal Aortic Aneurysms
}

\author{
Parveen Jayia, MRCS, Jason Constantinou, FRCS*, Hamish Hamilton, FRCS, \\ Krassi Ivancev, MD, PhD \\ Department of Vascular Surgery, Royal Free Hospital NHS Trust, London, United Kingdom
}

Based on a Presentation at the 2013 VEITH Symposium, November 19-23, 2013 (New York, NY, USA)

\begin{abstract}
Background: Spinal cord ischemia (SCI) is one of the most feared complications following the repair of thoraco-abdominal aortic aneurysms (TAAA). Endovascular repair of TAAA is now possible with branched stent grafts, but spinal cord ischaemia rates are still unacceptably high. A number of techniques have been utilized to reduce these levels, however, $\mathrm{SCI}$ remains a challenge to endovascular repair of TAAA. The use of sac perfusion branches aims to reduce the incidence of this catastrophic complication.

Methods: A retrospective analysis of all patients undergoing branched endovascular aortic repair for all thoraco-abdominalaneurysms(TAAA) using custom made devices during January 2008 to August 2014. We describe a two staged technique in which perfusion of segmental vessels is maintained by a temporary endoleak through an open perfusion branch, incorporated within the branched stent graft, followed by a closure of this branch at a later date to complete exclusion of the aneurysm.

Results: Forty-seven patients underwent TAAA repair. Twenty-five (53\%) had a two-stage procedure using either a sac perfusion branch or a target vessel to perfuse the sac. Nine patients $(19.15 \%)$ suffered some form of SCl with eight patients having temporary SCI (lasting less than 72 hours) and one patient having permanent $\mathrm{SCl}$. Of eight patients that had temporary spinal cord ischemia, all had a perfusion strategy. There was one case of permanent SCI (2.13\%).
\end{abstract}

Fax +1 2037853552

E-Mail:aorta@scienceinternational.org

http://aorta.scienceinternational.org
Conclusion: Sac perfusion branches provide a safe method for preventing $\mathrm{SCl}$, however this needs to be used in conjunction with controlling MAP and CSF drainage.

Copyright @ 2015 Science International Corp.

Key Words

Spinal cord ischaemia • Aneurysm • Perfusion • Thoraco-abdominal

\section{Introduction}

Spinal cord ischaemia $(\mathrm{SCl})$ resulting in complete paraplegia or paraparesis is a recognized complication during the treatment of thoracic or thoracoabdominal aortic aneurysms (TAAA). The symptoms can be apparent immediately (early $\mathrm{SCl}$ ) or present up to 72 hours later (delayed SCI) [1]. Open surgical and endovascular repair have been associated with $\mathrm{SCl}$, with some studies reporting incidences ranging between $11.4 \%$ to $22 \%$ [2]. The risk of SCl is increased by a number of factors such as the extent of the aneurysm and the corresponding stent graft length used and the number of posterior intercostal and lumbar arteries covered. There is much debate regarding the best intra-operative and post-operative adjuncts to prevent or minimise $\mathrm{SCl}$. Spinal cord protection strategies

\footnotetext{
* Corresponding Author:

Jason Constantinou, FRCS

Royal Free Hospital NHS Trust

London, UK

Tel: +44 0776692 8902, Fax: +44 0207472 6278, E-mail: Jason.constantinou@nhs.net
} 
have included adopting a 2-stage repair approach, reducing metabolic rate via induced hypothermia, measuring somatosensory-evoked potentials (SSEP) and motor-evoked potentials (MEP), monitoring cerebrospinal fluid (CSF) pressure, and reperfusion of intercostal arteries. A new and evolving technique is to maintain perfusion of intercostal and lumbar vessels by having an intentional endoleak [3]. This prevents complete thrombosis of the aneurysm sac, thereby maintaining spinal cord perfusion via the intercostals and lumbar arteries. We report our experience of using a two-staged technique in our high risk patients where either a temporary open branch incorporated into the main stent graft device or a target vessel is left open. These are then closed at a later date.

\section{Methods}

A two-center retrospective analysis of all patients undergoing branched endovascular aortic repair for all thoraco-abdominal aneurysms (TAAA) using custom made devices during January 2008 to August 2014. The team structure did not change and involved the same senior endovascular surgeons and senior interventional radiologists at both centers.

\section{Patients}

All patients with Type II-IV TAAA greater than $5.5 \mathrm{~cm}$, those who had a rapidly expanding aneurysm sac ( $>1 \mathrm{~cm}$ in 1 year), or those patients who were symptomatic were included in the study (Table 1). All patients were discussed at the local vascular multidisciplinary meeting prior to the procedure taking place.

\section{Endovascular Technique}

A two-staged approach was used for endovascular repair for type II-IV TAAA's that were deemed high risk for SCI. Each custom made Cook $^{\circledR}$ branched device had a temporary perfusion branch included in the design to create the temporary endoleak, or otherwise a target vessel (coeliac branch) was used as a perfusion branch (Figure 1). Each case was individually planned using TeraRecon 3D planning software.

In the first stage under general anesthesia, the custom made branched stent-graft was deployed and the branches connected to the visceral vessels. A sac perfusion branch was left open, allowing perfusion of the intercostal and lumbar vessels. A spinal drain was inserted. All patients were transferred to ITU post operatively where mean arterial pressure (MAP) was maintained above $90 \mathrm{mmHg}$ and spinal fluid was drained to ensure that the spinal cord pressure was lower than $10 \mathrm{~cm}$ of water. The management of spinal cord drains was standardized under a set institutional protocol. Patients were neurologically assessed hourly in ITU. Within a period of 3-months postoperatively, the patient underwent the second stage of the

\section{Results}

From 2008 to 2014, a total of 47 patients underwent TAAA repair. Of these, $25(53 \%)$ had a 2 -stage procedure using either a sac perfusion branch or a target vessel to perfuse the sac. Forty-five percent $(n=1)$ of the aneurysms were Type II TAAA.

Of the 25 patients, 19 (76\%) patients with Type II and 6 (24\%) with Type III TAAA aneurysms underwent 
sac perfusion with either a temporary branch or target vessel being left open. Perfusion strategies were not use in Type IV thoraco-abdominal aneurysms.

\section{Spinal Cord Ischemia (SCI)}

Nine patients (19.15\%) suffered some form of SCI with eight patients having temporary $\mathrm{SCl}$ (lasting less than 72 hours) and one patient having permanent $\mathrm{SCl}$. Five of the nine patients had perfusion branches that were closed in a second procedure and 3 patients had a target vessel deliberately left open (coeliac artery) that was successfully connected in a second procedure. Of eight patients that had temporary spinal cord ischemia, all had a perfusion strategy, and all recovered. There was one case of permanent $\mathrm{SCl}(2.13 \%)$. This patient had a Type II TAAA and did not have a perfusion branch as he was one of the early cases completed in the series.

Of the eight patients with temporary $\mathrm{SCl}$, seven had Type II TAAA and one occurred in a Type III TAAA.

There were no aneurysm ruptures during the interval from the first to second stage.

\section{Spinal Drain}

Forty-six patients had a spinal drain with most patients having a drain in for 2 days $(n=23)$. The remaining had a spinal drain in for 3 days $(n=20)$ and three patients had it only in for 24 hours.

\section{Mortality}

There was only one intra-operative death. This was in a patient who had a pre-operative contained thoracic rupture that decompensated hemodynamically intra-operatively.

There were five deaths (10.6\%) within 30 days. The causes of death were bowel ischaemia $(n=3)$, multi-organ failure $(n=1)$ and limb ischaemia $(n=1)$.

Six patients needed further surgical interventions following primary endovascular TAAA repair. The interventions ranged from laparotomies for bowel ischaemia $(n=3)$, lower limb salvage $(n=1$, fem-fem crossover, $n=1$ embolectomy) and one above-knee amputation for lower limb ischaemia.

\section{Discussion}

$\mathrm{SCl}$ is a recognized complication of TAAA repair. Due to the multi-factorial cause of $\mathrm{SCl}$, several strate- gies have been proposed.

In 1996, Safi described the importance of reimplanting intercostal arteries in conjunction with moderate hypothermia. This group prospectively analyzed 343 patients who had undergone either type I,II or III TAAA repair and showed that the benefit of reattachment was greatest for intercostals in the lower thoracic regions, in particular T9 through to T12 [4]. They also showed that greater benefit was seen for Type II TAAA, probably due to the greater aortic coverage, when combined with other spinal adjuncts such as spinal drainage. A series in 1993 by Svensson of 1509 patients showed that the greatest risk of paraplegia again was seen in Type II TAAA, where rates of $35 \%$ were reported. In this series, $45 \%$ $(n=684)$ had undergone reimplantation of intercostal arteries but it was difficult to identify how many were within the Type II TAAA group [5].

Monitoring motor evoked potentials (MEPS) has also been shown to be a reliable technique to assess spinal cord function intra-operatively during TAAA repair. In a study of 112 patients ( $n=70$ Type II TAAA), reduction in MEP correlated significantly with spinal cord ischaemia. However, even though it is a helpful tool to detect early SCI, MEP monitoring cannot guarantee prevention of delayed spinal cord ischemia [6]

A 2-staged repair has been shown in open surgery to be effective in reducing paraplegia rates. In a retrospective study of 90 patients, a 2-stage approach was effective with no $\mathrm{SCl}$ seen after the second stage even when there were a large number of intercostal/ lumbar arteries sacrificed [7].

We now use a 2-stage endovascular approach for all type II TAAAs whereby a temporary endoleak is created, allowing perfusion of the aneurysm sac and hence the spinal cord. This can be achieved either by the inclusion of a temporary perfusion branch or by simply leaving a target vessel open. In our series, nine patients (19.15\%) developed neurological symptoms following closure of the perfusion branch. The majority of these occurred in patients with Type II TAAA $(n=8)$ with the remainder $(n=1)$ occurring in Type III TAAA. Eight out of nine of these cases were temporary and resolved within 30 days. There was one patient with permanent disability in our series but this patient did not have sac perfusion (this was due to the fact that he was one of the 
first cases to have endovascular TAAA repair in the unit and the use of sac perfusion was not practiced). We believe that it is more important to stage cases and maintain sac perfusion as a concept, rather than specifically use perfusion branches. This could be achieved using various strategies, such as leaving the distal completion of stenting into the iliac arteries as a further stage, leaving a deliberate type 3 endoleak between undersized thoracic grafts for subsequent closure, or by delayed closure of a target visceral vessel branch.

Our findings suggest that maintaining sac perfusion to allow spinal cord perfusion is crucial in order to avoid the catastrophic complication of SCl. It is vital for high-risk patients to have spinal cord drainage and maintenance of an adequate MAP and avoidance of anemia [8].

One problem with our technique is that there remains a risk of aneurysm rupture due to the intentional temporary endoleak. In our small series of patients we did not see any aneurysm ruptures whilst waiting for the second stage of the procedure to be performed. If an aneurysm rupture were to occur we could simply repair this by percutaneous closure of the perfusion branches under local anesthesia. This technique can only be used in patients with an intact aortic sac and is contraindicated in those with a contained leak such as our patient that demised perioperatively. The efficacy of a temporary perfusion branch may be influenced by other parameters such as turbulent flow, shear stresses, or embolization, which paradoxically may induce spinal cord ischemia,

\section{Conclusion}

Controlled perfusion of the intercostal vessels with a temporary endoleak is feasible. Sac perfusion branches may be a useful adjunct to prevent $\mathrm{SCl}$, providing protection to spinal cord perfusion during the immediate post-operative period, when the risk of cardiovascular instability is greatest. As the general applicability of endovascular solutions to complex anatomical challenges increases, further innovations to prevent complications such as paraplegia are necessary. We believe that sac perfusion branches provide a safe method for preventing $\mathrm{SCl}$,
Table 1. Patient Characteristics and Risk Factors.

\begin{tabular}{|c|c|c|}
\hline Characteristic & Number & Percentage \\
\hline Mean Age (years) & $72.09(65-82)$ & \\
\hline Male & 32 & $68 \%$ \\
\hline Female & 15 & $32 \%$ \\
\hline Aneurysm Diameter $(\mathrm{cm})$ & 6.8 & \\
\hline \multicolumn{3}{|l|}{ Previous Aortic Repair: } \\
\hline None & 37 & $79 \%$ \\
\hline Open Aortic Repair & 6 & $13 \%$ \\
\hline Endovascular Repair & 4 & $8 \%$ \\
\hline \multicolumn{3}{|l|}{ No of branches: } \\
\hline 3 & 10 & $21 \%$ \\
\hline 4 & 36 & $77 \%$ \\
\hline 5 & 1 & $2 \%$ \\
\hline \multicolumn{3}{|l|}{ Cardiovascular Risk Factors: } \\
\hline Diabetes Mellitus & 5 & $6 \%$ \\
\hline Hypertension & 38 & $48 \%$ \\
\hline Smoking & 11 & $14 \%$ \\
\hline $\begin{array}{l}\text { Ischemic Heart } \\
\text { Disease }\end{array}$ & 17 & $21 \%$ \\
\hline Renal Failure & 9 & $11 \%$ \\
\hline \multicolumn{3}{|c|}{$\begin{array}{l}\text { American Society of } \\
\text { Anesthesiologists (ASA) Grade: }\end{array}$} \\
\hline 2 & 32 & $68 \%$ \\
\hline 3 & 12 & $26 \%$ \\
\hline 4 & 3 & $6 \%$ \\
\hline
\end{tabular}

however this needs to be used in conjunction with controlling MAP and CSF drainage. In the future, we plan to develop this model further to be used as a predictive tool as well as a protective tool. We believe that we will be able to perform "test-occlusions" of the branches whilst clinically monitoring neurological function and/or monitoring motor evoked potentials. If there is a disturbance in neurological function the branches need not be closed immediately, allowing time for development of collateral circulation.

\section{Conflict of Interest}

Krassi Ivancev is sponsored by COOK.

Comment on this Article or Ask a Question 


\section{References}

1. Ullery BW, Cheung AT, Fairman RM, Jackson BM, Woo EY, Bavaria J, et al. Risk factors, outcomes, and clinical manifestations of spinal cord ischaemia following thoracic endovascular aortic repair. J Vasc Surg. 2011;54:677684. DOI: 10.1016/j.jvs.2011.03.259

2. Böckler $D$, Kotelis $D$, Geisbüsch $P$, Hyhlik-Dürr A, Klemm K, von TenggKobligk $\mathrm{H}$, et al. Hybrid procedures for thoracoabdominal aortic aneurysm and chronic aortic dissections - a single center experience in 28 patients. J Vasc Surg. 2008;47:724-732. DOI: 10.1016/j. jvs.2007.12.009

3. Harrison SC, Agu O, Harris PL, Ivancev K. Elective sac perfusion to reduce the risk of neurologic events following endovascular repair of thoraco-abdominal aneurysms. J Vasc Surg. 2012;55:1202-1205. DOI: 10.1016/j.jvs.2011.10.079
4. Safi HJ, Miller CC, Carr C, lliopoulous D, Dorsay D, Baldwin JC. Importance of intercostal artery reattachment during thoraco-abdominal aortic aneurysm repair. J Vasc Surg. 1998;27:58-68. DOI: 10.1016/S07415214(98)70292-7

5. Svensson LS, Crawford S, Hus KR, Coselli JS, Safo HJ. Experience with 1509 patients undergoing thoracoabdominal aortic operations. J Vasc Surg. 1993;17:357-370. DOI: 10.1016/0741-5214(93)90421-H

6. Jacobs MJ, Mess W, Mochtar B, Nijenhuis RJ, Statius van Eps RG, et al. The value of motor evoked potentials in reducing paraplegia during thoraco-abdominal aneurysm repair. J Vasc Surg. 2006;43:239-246. DOI: 10.1016/j. jvs.2005.09.042

7. Etz CD, Zoli $S$, Mueller CS, Bodian CA, Luozzo GD, Lazala R, et al. Staged repair significantly reduces paraplegia rate after extensive thoraco-abdominal aortic aneurysm repair. J Thorac Cardiovasc Surg. 2010;139:1464-1472. DOI: 10.1016/j. jtcvs.2010.02.037

8. Huynh TT, Miller $3^{\text {rd }}$ CC, Safi HJ. Delayed onset of neurological deficit: Significance and management. Semin Vasc Surg. 2000;13:340-344. PMID: 11156063

Cite this article as: Jayia $\mathrm{P}$, Constantinou J, Hamilton H, Ivancev K. Temporary Perfusion Branches to Decrease Spinal Cord Ischemia in the Endovascular Treatment of Thoraco-Abdominal Aortic Aneurysms. AORTA 2015;3(2):5660. DOI: http://dx.doi.org/10.12945/j. aorta.2015.14-045 